\title{
Expression of carbonic anhydrase IX in
} human fetal joints, ligaments and tendons: a potential marker of mechanical stress in fetal development?

\author{
Ji Hyun Kim ${ }^{1}$, Seppo Parkkila ${ }^{2}$, Shunichi Shibata ${ }^{3}$, Mineko Fujimiya $^{4}$, Gen Murakami ${ }^{5}$, Baik Hwan Cho ${ }^{6}$ \\ ${ }^{1}$ Department of Anatomy, Chonbuk National University Medical School, Jeonju, Korea, ${ }^{2}$ Department of Anatomy, Institute of Bioscience and Medical \\ Technology, University of Tampere, Tampere, Finland, ${ }^{3}$ Maxillofacial Anatomy, Department of Maxillofacial Biology, Tokyo Medical and Dental University \\ Graduate School, Tokyo, ${ }^{4}$ Department of Anatomy, Sapporo Medical University School of Medicine, Sapporo, ${ }^{5}$ Division of Internal Medicine, Iwamizawa \\ Kojin-kai Hospital, Iwamizawa, Japan, ${ }^{6}$ Department of Surgery and Research Institute of Clinical Medicine, Chonbuk National University Hospital, \\ Chonbuk National University Medical School, Jeonju, Korea
}

\begin{abstract}
Carbonic anhydrase type IX (CA9) is known to express in the fetal joint cartilage to maintain pH against hypoxia. Using paraffin-embedded histology of 10 human fetuses at 10-16 weeks of gestation with an aid of immunohistochemistry of the intermediate filaments, matrix components (collagen types I and II, aggrecan, versican, fibronectin, tenascin, and hyaluronan) and CA9, we observed all joints and most of the entheses in the body. At any stages examined, CA9-poisitive cells were seen in the intervertebral disk and all joint cartilages including those of the facet joint of the vertebral column, but the accumulation area was reduced in the larger specimens. Glial fibrillary acidic protein (GFAP), one of the intermediate filaments, expressed in a part of the CA9-positive cartilages. Developing elastic cartilages were positive both of CA9 and GFAP. Notably, parts of the tendon or ligament facing to the joint, such as the joint surface of the annular ligament of the radius, were also positive for CA9. A distribution of each matrix components examined was not same as CA9. The bone-tendon and boneligament interface expressed CA9, but the duration at a site was limited to 3-4 weeks because the positive site was changed between stages. Thus, in the fetal entheses, CA9 expression displayed highly stage-dependent and site-dependent manners. CA9 in the fetal entheses seemed to play an additional role, but it was most likely to be useful as an excellent marker of mechanical stress at the start of enthesis development.
\end{abstract}

Key words: Carbonic anhydrase type IX, Intermediate filaments, Joints, Enthesis, Human fetus

Received August 8, 2013; 1st Revised September 5, 2013, 2nd Revised September 6, 2013; Accepted September 27, 2013

\section{Introduction}

It is well known that mechanical stresses, such as tensile, compression and shear stresses, determine and maintain

\author{
Corresponding author: \\ Baik Hwan Cho \\ Department of Surgery, Chonbuk National University Medical School, \\ 20 Geonji-ro, Deokjin-gu, Jeonju 561-712, Korea \\ Tel: +82-63-250-1570, Fax: +82-63-271-6197, E-mail: chobh@jbnu.ac.kr
}

the morphology of the musculoskeletal system during fetal development [1-4]. In essence, early muscle contraction "teaches" a specific morphology to the tendon, which, in turn, teaches a specific shape to the bone. The matrix components of entheses (bone-tendon and bone-ligament interfaces), such as collagen type I and II, aggrecan, versican, fibronectin, tenascin and hyaluronan, change depending on the strength and modality of the mechanical stress [5]. In addition to matrix components, mechanical loading of the temporomandibular joint disk in an experimental rat model 
was shown to change the expression of intermediate filaments (10-nm filaments), glial fibrillary acidic protein (GFAP), and desmin [6]. Desmin plays a critical role in the initial attachment between striated muscle and tendons [7] and is thought to maintain the stability of mesenchymal cells in association with vimentin, another intermediate filament [8] However, the interest in GFAP appears to have been limited to a consideration of its role in elastic cartilage development $[9,10]$. To our knowledge, the expression of intermediate filaments in human fetal entheses has not yet been extensively examined.

Another major player in the current study that has received less research attention in the context of fetal enthesis development than matrix components is carbonic anhydrase (CA). CAs are zinc metalloenzymes that catalyze the reversible hydration of $\mathrm{CO}_{2}$ into bicarbonate and hydrogen ions. More than 15 isoenzymes of CAs are found in cellular secretions, cytosol, mitochondria, or bound to the plasma membrane. CAs have broad biological functions, including the regulation of $\mathrm{pH}$, removal of metabolic waste, transportation of ions across the plasma membrane, gluconeogenesis, lipogenesis, urea genesis, bone resorption, and calcification [11-13]. Carbonic anhydrase IX (CA9), a transmembrane CA isoenzyme with a proteoglycan domain, is predominantly expressed in human tumors in response to hypoxia and has been functionally implicated in the adaptation of tumor cells to hypoxic stress via control of $\mathrm{pH}$ and cell adhesion $[14,15]$. Notably, in contrast to other CAs isoenzymes, CA9 expression has been reported in cartilage, tendons, ligaments, and striated muscle [16-18].

Most reports of CA9 expression in the musculoskeletal system have centered on its presence in the intervertebral disks of the vertebral column and joints of the extremities. In contrast, little or no research attention has been paid to CA9 expression at entheses. The early development of entheses takes place at a time when there are few or no blood vessels and thus is likely to occur under extremely hypoxic conditions. CA9, which is first detected in the fetal musculoskeletal system at 7 weeks of gestation [17], is likely to specifically play a role in maintaining the fetal microenvironment in which the enthesis develops. To provide a better understanding of human fetal development of entheses, we sought to clarify the spatial and temporal relationships between the expression of matrix proteins and intermediate filaments and that of CA9. We examined these relationships in specimens from fetuses at 10-16 weeks gestation, a time when CA9 expression has previously been reported to be strongest [17].

\section{Materials and Methods}

The study was performed in accordance with the provisions of the Declaration of Helsinki 1995 (as revised in Edinburgh 2000). The histology of paraffin-embedded specimens was examined in a total of 10 mid-term fetuses at estimated gestational ages of 10-16 weeks (crown-rump length [CRL], 50-120 mm): two from 10-week fetuses (CRL, 50-58 mm), three from 12-week fetuses (CRL, 71-80 $\mathrm{mm}$ ), and five from 15-16-week fetuses (CRL, 102-120 $\mathrm{mm}$ ). These specimens were donated to the Department of Surgery, Chonbuk National University, Korea, with the agreement of the subjects' families, and their use for research was approved by the Chonbuk National University Ethics Committee. Under applicable rules and regulations, the approval of the corresponding Japanese Committee was not required. The fetuses were obtained from patients undergoing induced abortions. After the procedure, each mother was personally informed of the possibility of donating the fetus for research by an obstetrician; no attempt was made to encourage donation. Specimen numbers were randomized, making it impossible to trace specimens to any of the families concerned.

The donated fetuses were fixed with $10 \%(\mathrm{w} / \mathrm{w})$ neutral formalin solution for more than 1 month. After dividing into head and neck, thorax, abdomen, pelvis and the four extremities, samples were decalcified by incubating at $4^{\circ} \mathrm{C}$ in a $0.5-\mathrm{mol} / \mathrm{l}$ ethylenediaminetetraacetic acid ( $\mathrm{pH} 7.5)$ solution (decalcifying solution B, Wako, Tokyo, Japan) for 1-3 days, depending on the size of the sample. The scapula and its associated muscles were included in the thoracic segment, and the hip joint and its associated muscles were included in the pelvis segment. Specimens for histological analysis were sectioned ( $5 \mu \mathrm{m}$ thickness) and paraffin embedded using routing procedures. Samples were sectioned at 20-100 $\mu \mathrm{m}$ intervals, depending on sample size. Head and pelvic segments were sectioned sagittally or horizontally; abdominal segments were sectioned sagittally; thoracic segments were sectioned horizontally; and the extremities were sectioned longitudinally along the proximodistal axis. Most sections were stained with hematoxylin and eosin; some were reserved for immunohistochemistry.

Two primary antibodies were used for immunohisto- 
chemical detection of CA9: 1) a rabbit polyclonal anti-human CA9 (1:500), originally established by a group organized by the second author [19]; and 2) a commercially available rabbit polyclonal anti-human CA9 (1:1,000, ab15086, Abcam, Cambridge, UK). The latter antibody was used to provide a convenient reference for others seeking to reproduce our work. The following primary antibodies were used for immunohistochemical detection of intermediate filaments: 1 ) rabbit polyclonal anti-human GFAP (1:100, Z0334, Dako Cytomation, Kyoto, Japan), 2) mouse monoclonal anti-human desmin (1:100, M0760, Dako, Glostrup, Denmark), 3) mouse monoclonal anti-human vimentin (1:10, M7020, Dako), 4) mouse monoclonal anti-human smooth muscle alpha actin (SMA; 1:100, M0851, Dako), and 5) mouse monoclonal antihuman nestin (1:50, sc23927, Santa Cruz Biotechnology, Santa Cruz, CA, USA). Samples were not pretreated by autoclaving (antigen retrieval) because of the loose nature of the fetal tissues. After incubating with primary antibodies at $4^{\circ} \mathrm{C}$ for overnight, samples were incubated for 30 minutes at room temperature. with horseradish peroxidase (HRP)conjugated secondary antibody (Histofine Simple Stain MaxPO, Nichirei, Tokyo, Japan), and immunoreactive proteins were detected by monitoring the HRP-mediated catalysis of the substrate diaminobenzidine (3-5-minute incubation at room temperature, Histofine Simple Stain DAB, Nichirei). All samples were counterstained with hematoxylin. Negative controls consisted of samples without primary antibody. The Dako anti-SMA antibody strongly reacts with not only smooth muscle cells but endothelium of arteries and veins [20].

The matrix components collagen types I and II, aggrecan, versican, fibronectin, tenascin and hyaluronan, as an essential minimal set [21], were also detected by immunohistochemistry as we have done previously [22-24]. Hyaluronan was stained using biotinylated hyaluronan binding protein $(2 \mu \mathrm{g} / \mathrm{ml}$, Seikagaku Corp., Tokyo, Japan) after immersion of sections in chondroitinase $\mathrm{ABC}(0.01 \mathrm{units} / \mathrm{ml}$, Seikagaku Corp.) in $0.1 \mathrm{M}$ Tris-acetate buffer ( $\mathrm{pH}$ 8.0) for 30 minutes at $37^{\circ} \mathrm{C}[22]$. For immunohistochemical detection of other matrix components, samples were pretreated with testicular hyaluronidase $(25 \mathrm{mg} / \mathrm{ml}$, Sigma Chemicals, St. Louis, MO, USA) in phosphate buffered saline at $37^{\circ} \mathrm{C}$ for 30 minutes. The following primary antibodies were used: 1 ) rabbit polyclonal anti-rat type I collagen (1:400, LSL, Tokyo, Japan), 2) rabbit polyclonal anti-rat type II collagen (1:400, LSL), 3) mouse monoclonal anti-aggrecan core protein (1:25,
12/21/1C6, Developmental Studies Hybridoma Bank, Iowa City, IA, USA), 4) mouse monoclonal anti-versican core protein $(1: 25,12 \mathrm{C} 5$, Developmental Studies Hybridoma Bank), 5) rabbit polyclonal ant-rat fibronectin (1:400, LSL), and 6) rabbit polyclonal ant-rat tenascin-C (1:100, Chemicon, Temecula, CA, USA). All sections were treated with testicular hyaluronidase (Sigma type I-S, Sigma Chemicals) before challenging with primary antibodies. After incubation with secondary antibody (Histofine SAB kit, Nichirei), the sections were treated with 3-amino-9-ethylcarbazole (AEC), resulting in the formation of red immune complexes. Negative controls consisted of samples incubated without primary antibody.

\section{Results}

\section{Distribution of CA9-positive cells}

CA9-positive cells were concentrated in the developing intervertebral disks of the vertebral column, including the anulus pulposus (Fig. 1), at all levels from the axis to the coccyx. The facet joints of vertebrae as well as joint capsules were also positive at all levels (Fig. 1), as were cartilage surfaces at the occipito-vertebral junction (data not shown). There were no differences in immunoreactivity in intervertebral disks or facet joints among specimens examined. At the occipitovertebral junction, transverse atlantis and alar ligaments were positive for CA9 at 10-12 weeks, but were very weak at 15-16 weeks (data not shown).

In addition to the vertebral column, we examined virtually all joints in the body. CA9 was expressed in all surface cartilages facing the joint cavity as well as in all joint disks. Among the CA9-positive disks were the meniscus of the knee (Fig. 3) and an irregularly shaped disk at the wrist known as the triangular fibrocartilage complex (Fig. 5) or triangular fibrocartilage complex [25]. The articular cartilage was not clearly discriminated from other cartilages owing to its poor differentiation. However, parts of the cartilage distant from the joint, such as the shaft or body, were negative (Fig. 4). CA9 expression was weak in intertarsal and intercarpal joints (Figs. 2, 5). As shown in Fig. 2, which depicts the glenohumeral, hip, ankle and temporomandibular joints of pairs of smaller and larger specimens, positive areas in the glenohumeral, hip, and ankle joints became restricted in the larger specimens (Fig. 2B, $\mathrm{D}, \mathrm{F}$ ), indicating a decline in CA9 expression with increasing fetal age. Evidence for stronger expression at an earlier gestation time was also seen in other joints of the extremities, including the sternoclavicular and acromioclavicular joints. 


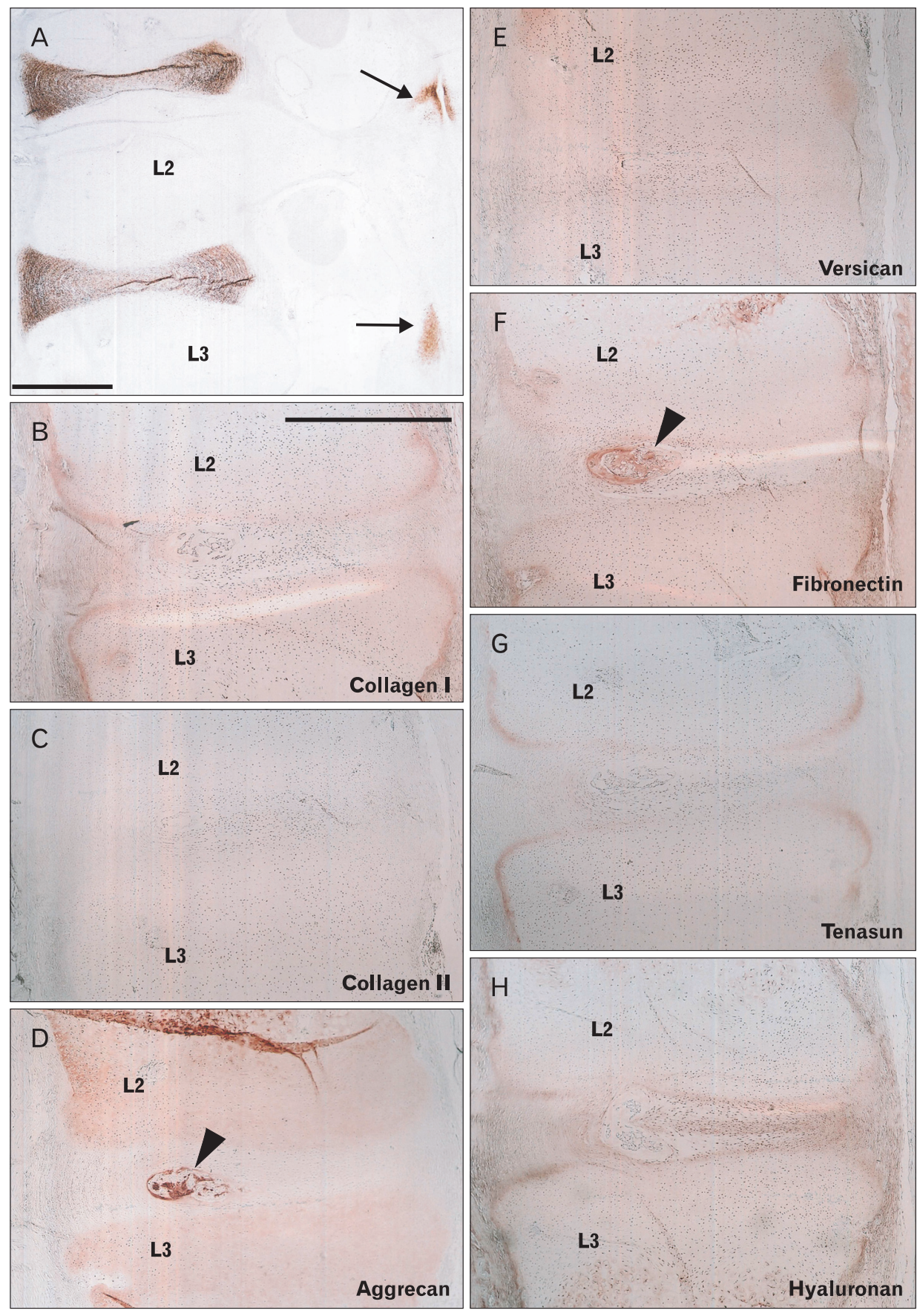

Fig. 1. Intervertebral disk of a 16-week fetus. Sagittal sections; second and third lumbar vertebrae levels (L2, L3). The left-hand side of each panel corresponds to the posterior side of the body. Immunohistochemical detection of carbonic anhydrase IX (CA9) (A), collagen type 1 (B), collagen type 2 (C), aggrecan $(D)$, versican $(E)$, fibronectin $(F)$, tena$\operatorname{scin}(\mathrm{G})$, and hyaluronan $(\mathrm{H})$. CA9 expression is restricted in the intervertebral disk and facet joint (arrows in panel A). No matrix protein is specific for the intervertebral disk, although the primitive nucleus pulposus (arrowheads in panels $\mathrm{D}$ and $\mathrm{F}$ ) is strongly positive for aggrecan and fibronectin. Panels $(\mathrm{B}-\mathrm{H})$ are presented at the same magnification. Scale bars in panels (A) and $(B)=1 \mathrm{~mm}(\mathrm{~A}), 1 \mathrm{~mm}(\mathrm{~B}-\mathrm{H})$.
Likewise, CA9 immunoreactivity was weaker in the larger, older specimen in the trachea and larynx (specifically in the tracheal cartilage rings), the cricoarytenoid joint of the larynx and an attachment between the thyroid cartilage and the greater horn of the hyoid bone (Fig. 6). Conversely, in the larger specimens, CA9 immunoreactivity increased in area in the temporomandibular joint (Fig. $2 \mathrm{H}$ ) and the sacroiliac joint. However, CA9 immunoreactivity was absent in the smaller specimens and was weak in the larger specimens in articulations of ear ossicles (incudomalleolar, incudostapedial, and stapediovestibular joints) as well as in auricular and pharyngotympanic tube cartilages (data not shown).

Notably, CA9 immunoreactivity was detected in tendons, ligaments, and other muscle-associated tissues, and displayed some site- and stage-dependence. In the vertebral column, the attachment of the interspinous ligament to the spinous process 

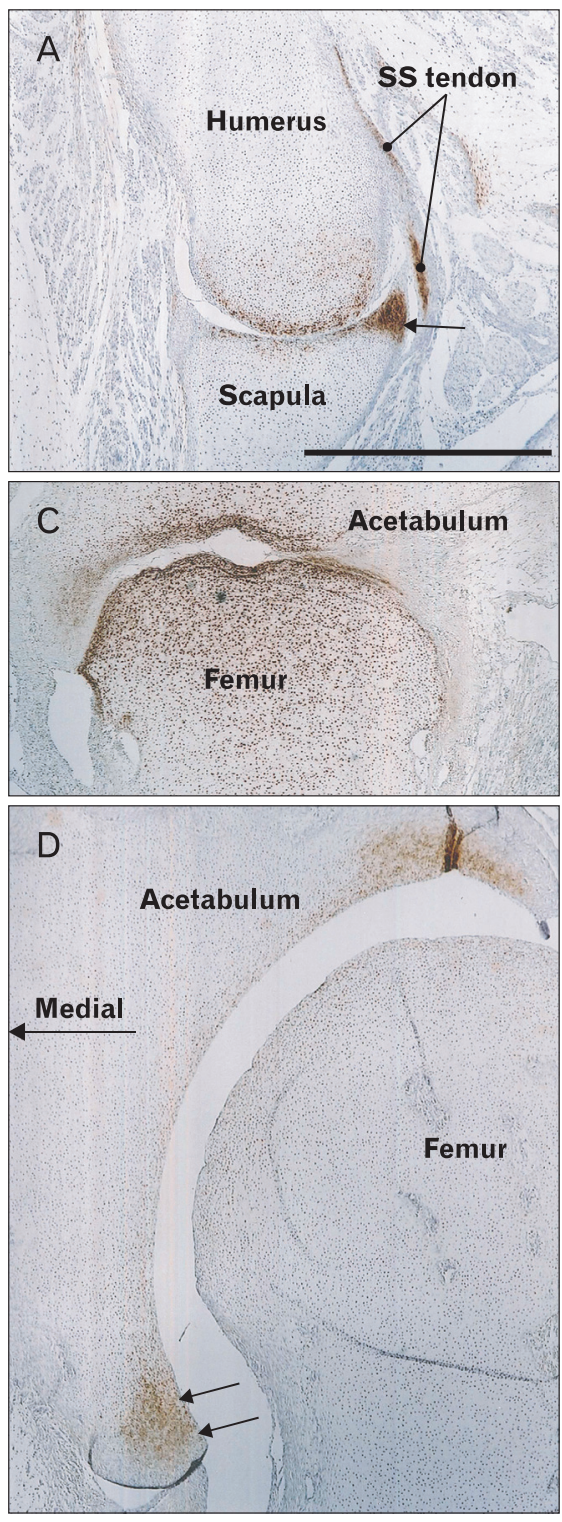

G Temporal bone

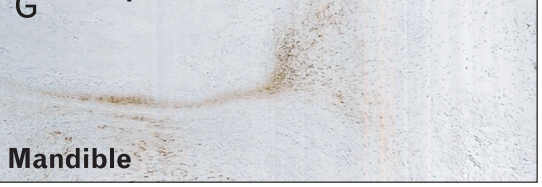

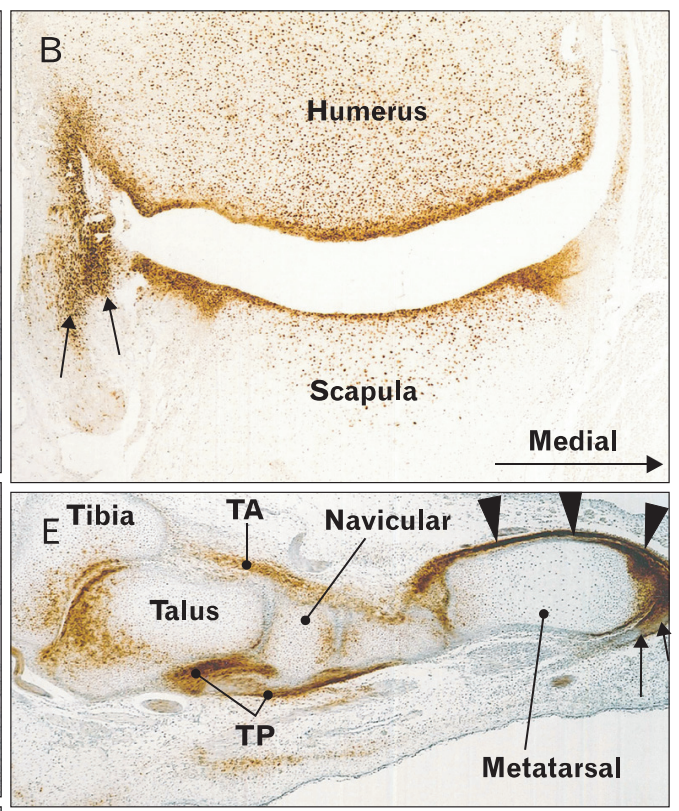

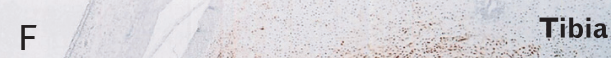

FDL
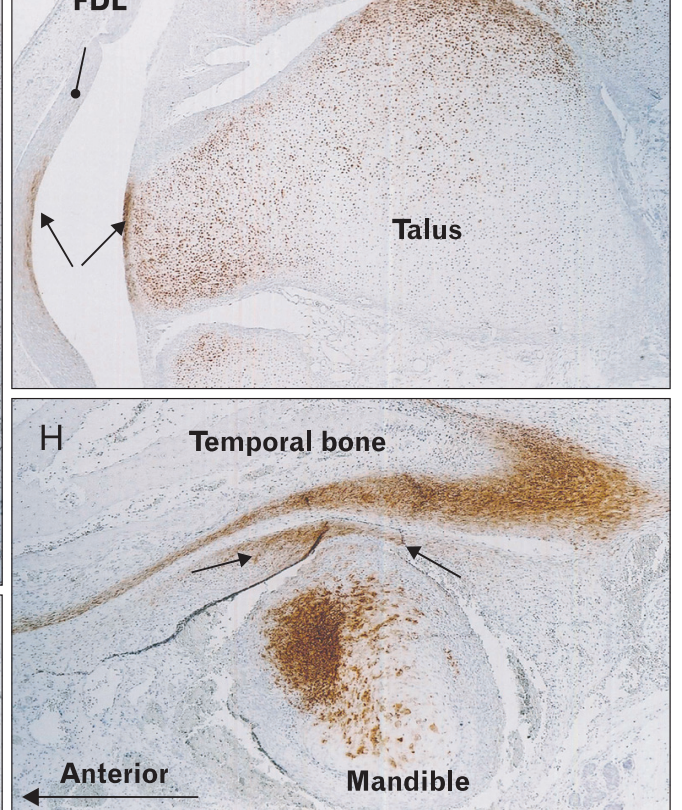

Fig. 2. Carbonic anhydrase type IX (CA9) expression in joint surfaces and associated ligaments and tendons. Immunohistochemical detection of CA9. Panel (A; 11 weeks) and panel (B; 15 weeks) show the glenohumeral joint (horizontal sections). The lip of the glenoid fossa (arrow in panels $\mathrm{A}$ and $\mathrm{B}$ ) as well as the tendon of the subscapularis muscle (SS tendon in panel A) are strongly positive. Panel (C; 12 weeks) and panel (D; 16 weeks) show the hip joint (horizontal sections); the lip of the acetabulum is positive (arrows in panel D). Panel (E; 11 weeks) and panel (F; 16 weeks) show the talocrural joint (longitudinal sections). The tendons of the tibialis anterior and posterior (TA, TP), extensors (arrowheads), and flexors (arrows) are positive (E), whereas the tendon of the flexor digitorum longus (FDL) and its corresponding talus surface (arrows) are positive (F). Panel ( $\mathrm{G} ; 10$ weeks) and panel $(\mathrm{H} ; 16$ weeks $)$ show the temporomandibular joint (sagittal sections); the disk is positive in the larger specimen (arrows in panel $\mathrm{H})$. All panels are presented at the same magnification. Scale bar in panel $(A)=1$ $\mathrm{mm}(\mathrm{A}-\mathrm{H})$. was positive at all stages examined (data not shown). In the shoulder, the lip of the glenoid fossa and the insertion tendon of the subscapularis were strongly positive in the smaller specimens (Fig. 2A). The latissimus dorsi muscle insertion to the humerus was also positive. In contrast, the common insertion of the supra- and infraspinatus muscles was negative (data not shown). However, intramuscular tendons of the infraspinatus were positive. The lip of the acetabulum was positive in the larger specimens (Fig. 2D). In the hand and foot of smaller specimens, insertions of the long extensor and flexor tendons to the finger bones were positive (Fig. 2E). Likewise, tendons of the tibialis anterior and posterior muscle were also positive at the insertion to the navicular and other tarsal bones (Fig. 2E). However, the long tendon of the peroneus communis muscle as well as the calcaneal tendon was negative. In the extremities of larger specimens, we found strong expression of CA9 in the quadriceps tendon insertion to the patella (Fig. 3), the annular ligament facing the 
circumferential articular surface of the head of the radius (Fig. 4), and the intercarpal interosseus ligament of the hand (Fig. 5). However, we found no expression in insertion tendons of the triceps brachii, biceps brachii or brachialis muscles, or in any collateral ligaments of the extremities (data not shown).

Fig. 6 shows CA9-positive structures distant from the joint in the larger specimens. These include a ligamentous tissue (possibly a thyroepiglottic ligament) from the thyroid cartilage (Fig. 6A), the medial collateral ligament between the conjunctiva and the medial rectus muscle of the eye
(Fig. 6B), the insertion of the rectus abdominis muscle to the pubis (Fig. 6C), the linear aspera of the femur (Fig. 6D), the ischial tuberosity (Fig. 6E), and the inguinal ligament (Fig. 6F). The origin of the rectus femoris in the ilium was also positive. Although the midline septum of the rectus abdominis (the putative linea alba) was positive, the rectus sheath was negative. Another paradoxical set of findings was the observation that the aponeurosis for the rhomboideus muscle origin was CA9-positive, whereas the aponeurotic origin of the trapezius muscle in the back of the upper thorax

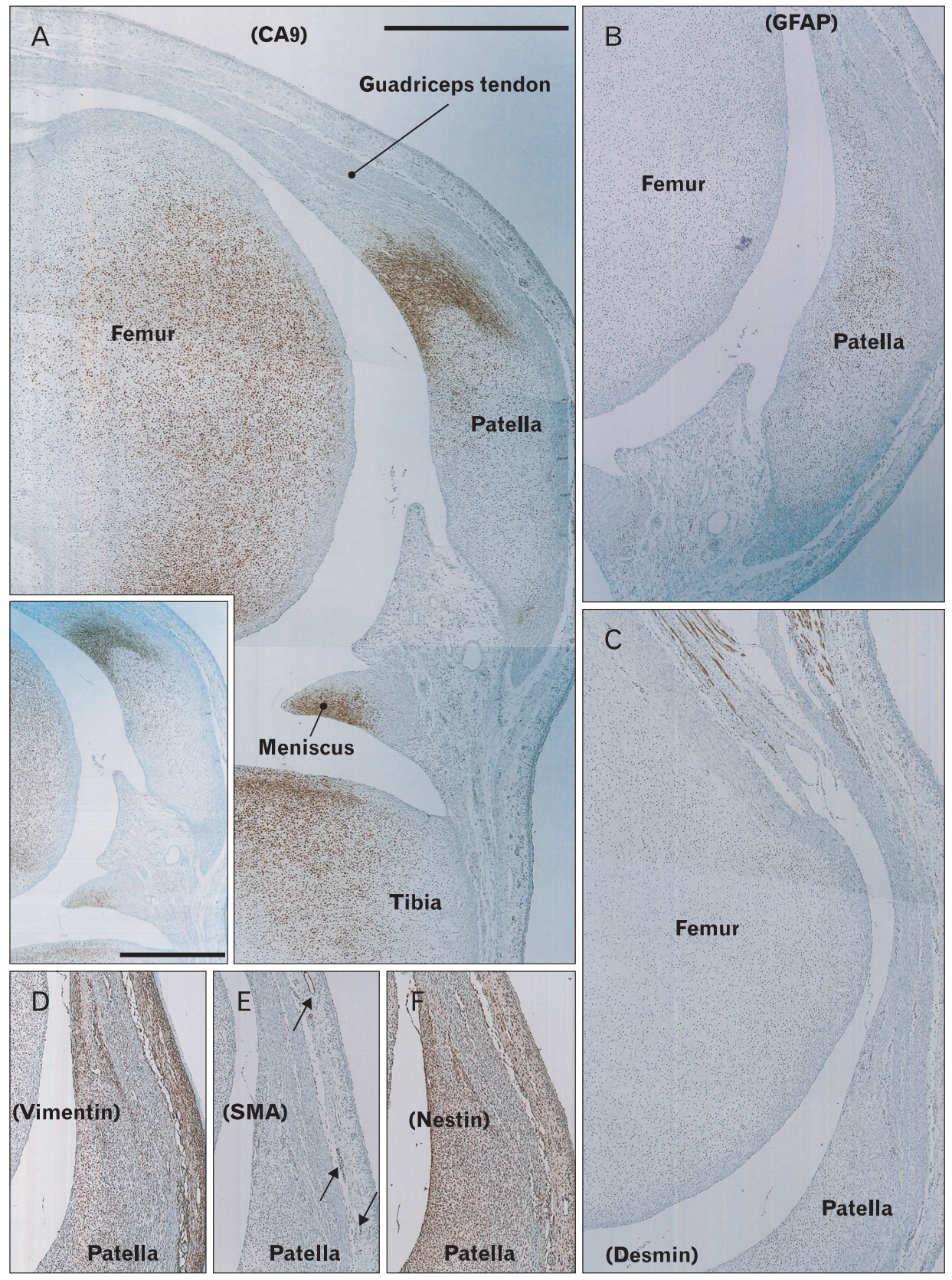

Fig. 3. Expression of carbonic anhydrase type IX (CA9) and intermediate filaments in the knee of a 15 -week fetus. Longitudinal sections. Immunohistochemical detection of CA9 (A), glial fibrillary acidic protein (GFAP) (B), desmin (C), vimentin (D), smooth muscle alpha actin (SMA) (E), and nestin (F). The commercial antibody was used only for the inset in panel (A). (A) CA9 is strongly expressed at the interface area between the quadriceps muscle tendon and the patella. (B) Abundant GFAPpositive cells are seen in the patella, but few are present in the interface and tendon. (D, F) Vimentin and nestin are diffusely expressed in the connective tissue. The anti-SMA antibody strongly stains vascular smooth muscle cells (arrows in panel E). All panels show nearby sections and are presented at the same magnification, except for the inset in panel (A). Scale bar in panel (A) and inset $=1 \mathrm{~mm}$ (A-F), $1 \mathrm{~mm}$ (inset). 
was CA9-negative. In all of these structures, the intraorbital ligaments could clearly be distinguished from others because they were not connected to a hard tissue. In addition, CA9 immunoreactivity was strong in the pulley (cartilage and tendon) of the superior oblique muscle of the eye as well as in the tendon of the tensor veli palatini muscle around the hamulus of the pterygoid process at all stages examined (data not shown).

There were some difference in CA9 immunoreactivity in striated muscles detected using the original antibody developed in our laboratory [19] and the commercial antibody. We found that the muscles were strongly positive using the latter antibody, but were weakly positive or negative using the antibody developed by us. However, the skin dermis, oral epithelium, pleura, pericardium, and peritoneum were strongly positive using the original antibody. With the exception of an insert in Fig. 3A, all figures showing CA9 expression are based on results obtained using the original antibody.

\section{Intermediate filaments and CA9}

Using sections near those used for immunohistochemical detection of CA9, we observed intermediate filaments immunoreactivity in the vertebral column, skull base, eye, neck, knee (Fig. 3), foot, elbow (Fig. 4), and wrist (Fig. 5). Outside of nerve tissues, GFAP was detected in surface cartilage facing the joint cavity rather than in tendons or ligaments. Some positive cells were seen in the quadriceps muscle insertion to the patella (Fig. 3B). However, there were fewer of these GFAP-positive cells than CA9-positive cells at the same site. In the intervertebral disk, only a few cells were positive for GFAP; in contrast, these cells strongly expressed CA9. The articular disk, including the meniscus of the knee, also contained a few GFAP-positive cells (Figs. $3 \mathrm{~B}, 5 \mathrm{~B}$ ). In the elbow, the head of the radius was positive for GFAP, but the annular ligament was negative (Fig. 4B). GFAP immunoreactivity in the tendon insertion was much weaker than that of CA9 at all sites examined. The areas along and around the laryngeal cartilages and hyoid bone were rich in GFAP-positive mesenchymal cells. Overall, the distribution of GFAP-positive cells was similar to that of CA9-positive cells, but the frequency of these cells and the intensity of their staining were decreased compared with CA9-positive cells.

All striated muscles were positive for desmin, but we found
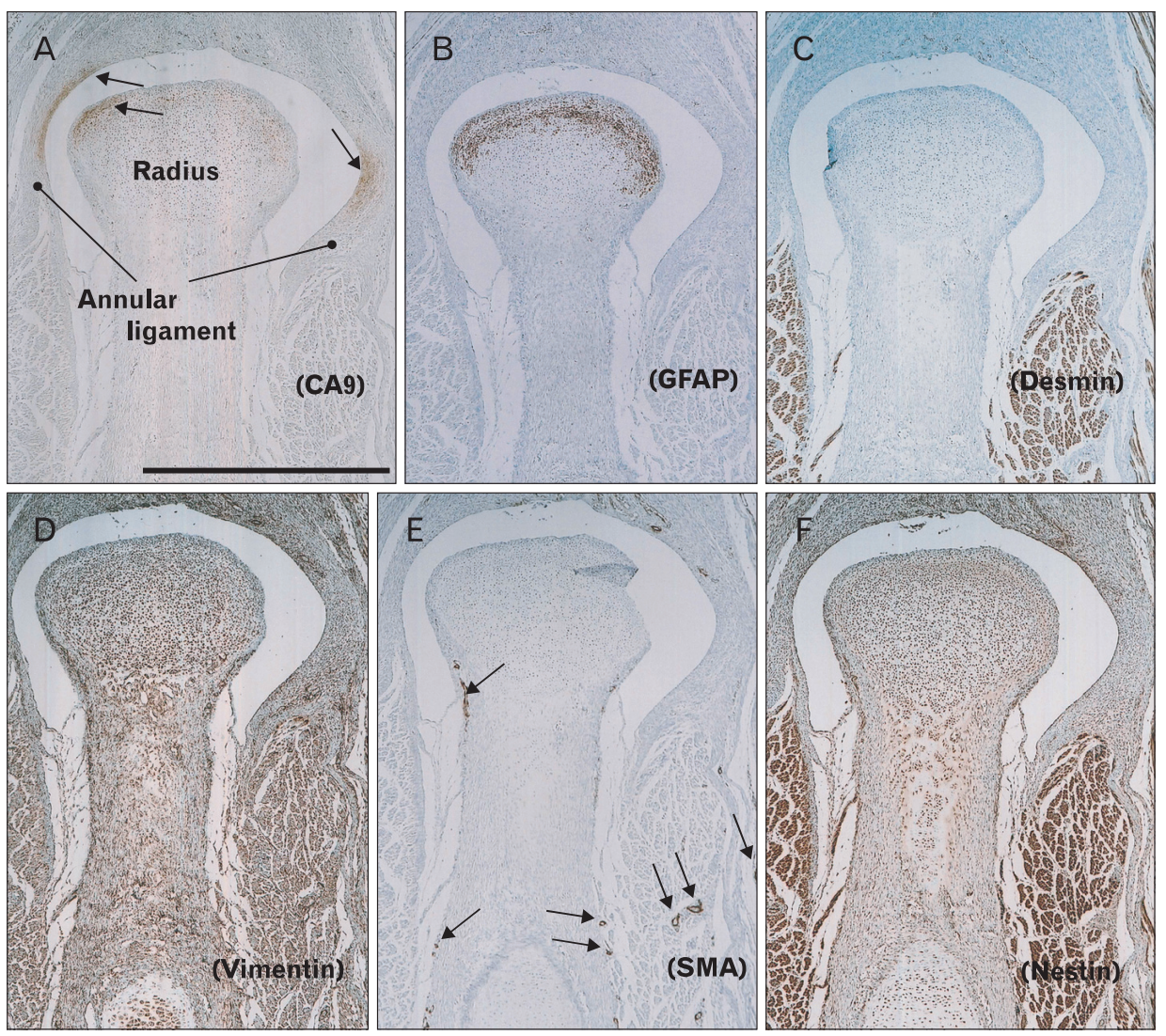

Fig. 4. Expression of carbonic anhydrase type IX (CA9) and intermediate filaments in the elbow of a 14 week fetus. Longitudinal sections. Immunohistochemical detection of CA9 (A), glial fibrillary acidic protein (GFAP) (B), desmin (C), vimentin (D), smooth muscle alpha actin (SMA) (E), and nestin (F). CA9 expression is detected not only at the head of the radius, but also at surfaces where the annular ligament is attached (arrows in panel A). (B) GFAP-positive cells are detected at the head of the radius, but not in the annular ligament. (D, F) Vimentin and nestin are diffusely expressed in connective tissue. The antiSMA antibody strongly stains vascular smooth muscle cells (arrows in panel E). All panels are nearby sections and are presented at the same magnification. Scale bar in panel $(A)=1 \mathrm{~mm}(A-F)$. 

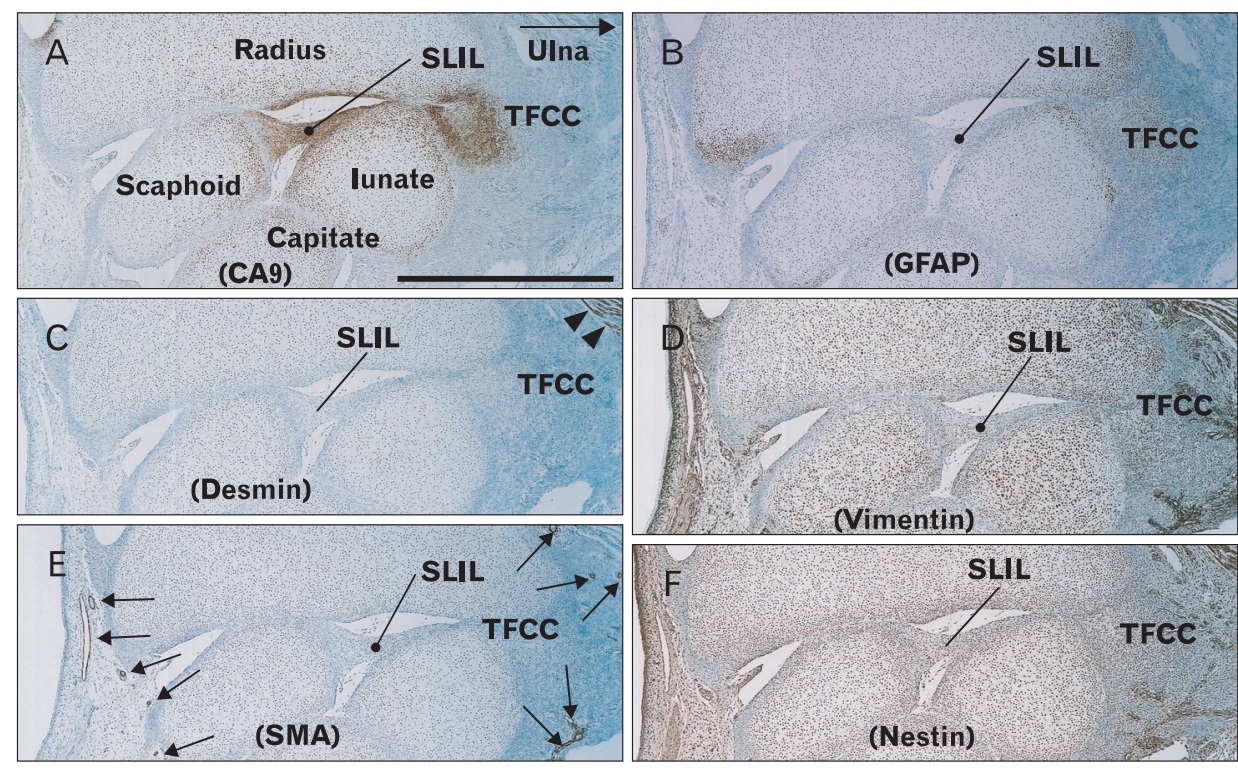

Fig. 5. Expression of carbonic anhydrase type IX (CA9) and intermediate filaments in the wrist of a 16-week fetus. Longitudinal sections. Immunohistochemical detection of CA9 (A), glial fibrillary acidic protein (GFAP) (B), desmin (C), vimentin (D), smooth muscle alpha actin (SMA) (E), and nestin (F). (A) CA9 is expressed not only at the joint surface of the carpal bones, but also in the scapholunate interosseus ligament (SLIL) and the triangular fibrocartilage complex or triangular fibrocartilage complex (TFCC). (B) GFAP-positive cells are detected in carpal bones, but not in the ligament. (C) Desmin is expressed in a portion of the pronator quadrates muscle (arrows) on the ulnar side. (D, F) Vimentin and nestin are diffusely expressed in connective tissue, including the ligament. The anti-SMA antibody strongly stains vascular smooth muscle cells (arrows in panel E). All panels are nearby sections and are presented at the same magnification. Scale bar in panel $(\mathrm{A})=1 \mathrm{~mm}(\mathrm{~A}-\mathrm{F})$.

no desmin immunoreactivity in the joint, tendon, or ligament (Figs. 3C, 4C, 5C). Vimentin-positive cells were distributed in all connective tissue, including osteoprogenitor cells and skin dermis (Figs. 3D, 4D, 5D). All arteries and veins were positive for SMA (Figs. 3E, 4E, 5E), but CA9-positive regions were negative for SMA staining, clearly demonstrating the absence of blood supply in these areas. Overlapping SMA and CA9 expression was limited to a connective tissue band extending along the anteroposterior axis in the superior side of the eyeball that appeared to correspond to the capsulopalpebral ligament [26]. The collateral ligaments of the eye were negative for SMA. The distribution of nestin immunoreactivity (Figs. 3F, 4F, 5F) was similar to that of vimentin. However, in contrast to vimentin, nestin was strongly expressed in striated muscle fibers. Overall, intermediate filaments other than GFAP exhibited distribution patterns quite different from those of CA9.

\section{Matrix components and CA9}

In sections near those used for immunodetection of CA9, we observed immunoreactivity for matrix components in the vertebral column (Fig. 1); skull base, including the middle ear and temporomandibular joint; neck; shoulder; hip; and foot (Fig. 7). In the vertebral column, collagen type 1 (Fig. 1B) and tenascin (Fig. 1G) immunoreactivity were distributed along cartilage surfaces facing the intervertebral disk. The nucleus pulposus strongly expressed aggrecan (Fig. 1D) and fibronectin (Fig. 1F). However, no matrix components examined were specifically positive in the disk or facet. In the temporomandibular joint, no aggrecan or tenascin immunoreactivity was detected on the surface of the condyle of the mandible. The disk was positive for collagen type I, versican, and fibronectin. In the foot, versican immunoreactivity was detected along finger bone surfaces, but not in the tendon insertion (Fig. 7D). Tenascin immunoreactivity was also observed along tarsal bone surfaces, but not in the joint surface (Fig. 7F). Overall, all matrix components examined showed a distribution pattern different from that of CA9.

\section{Discussion}

\section{Matrix substances and CA9}

When this study was conceived, we hypothesized that a specific matrix component and/or some intermediate filaments, especially GFAP, would be expressed in areas where 


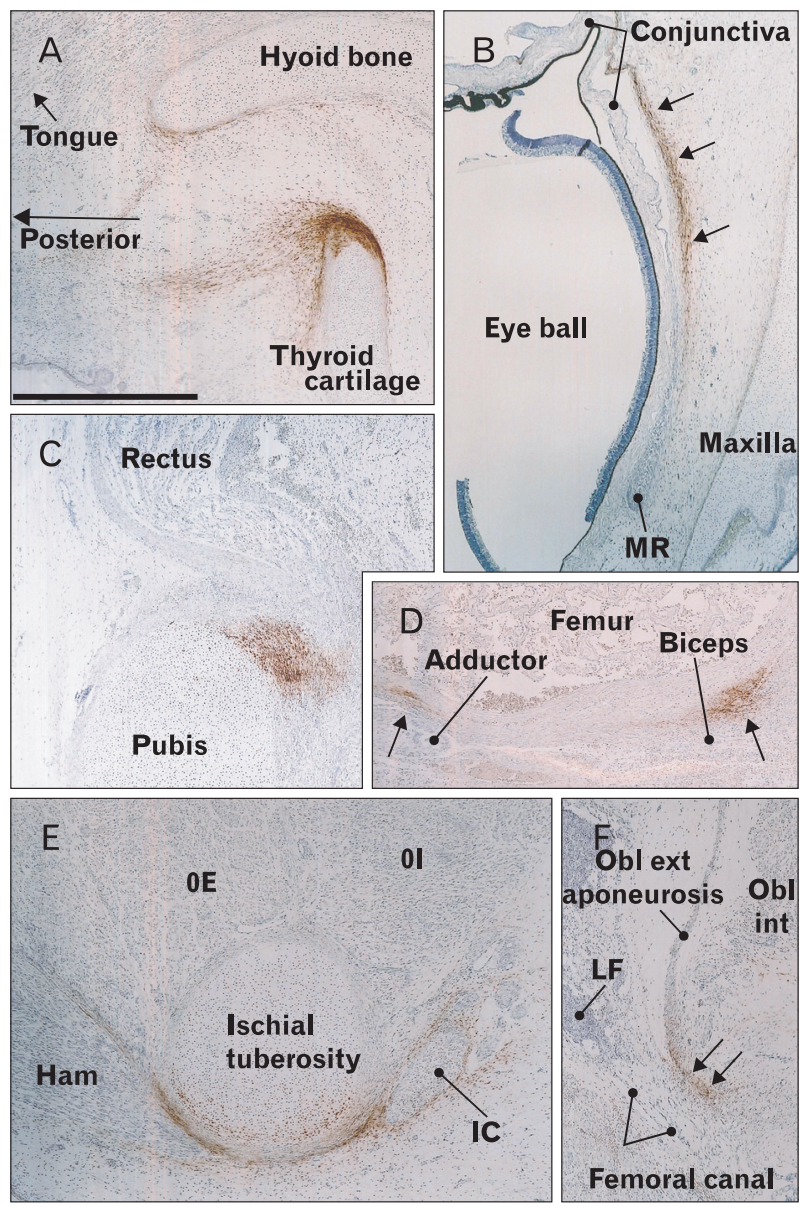

Fig. 6. Carbonic anhydrase type IX (CA9)-positive connective tissues distant from joints. Immunohistochemical detection of CA9. Panel (A; sagittal section, 16 weeks) shows CA9-positive ligamentous tissue from the thyroid cartilage. Panel (B; sagittal section, 14 weeks) shows a portion of the eye containing the medial collateral ligament (arrows) that connects between the medial rectus muscle (MR) and conjunctiva. Panel (C; sagittal sections, 15 weeks) shows the insertion of the rectus abdominis muscle (rectus) to the pubis. Panel (D; horizontal section, 16 weeks) shows the linear aspera of the femur in which CA9 expression (arrows) is detected at attachments of both adductor magnus (adductor) and biceps femoris (biceps) muscles. Panel (E; sagittal, 15 weeks) shows the ischial tuberosity to which the hamstrings (Ham) and ischiocavernosus muscle (IC) attach. Panel (F; sagittal, 12 weeks) shows the inguinal ligament (arrows) that faces the femoral canal and connects with the insertion aponeurosis of the obliquus externus muscle (obl ext aponeurosis) and the obliquus internus muscle (obl int). LF, lymphatic follicle; OE, obturator externus muscle; OI, obturator internus muscle. All panels are presented at the same magnification. Scale bar in panel $(A)=1 \mathrm{~mm}(\mathrm{~A}-\mathrm{F})$.

CA9-positive cells accumulated. However, CA9-positive joint cartilage and intervertebral disks contained no specific matrix proteins. Except for the expression of tenascin and versican in tarsal bones, there was not a clear margin between positive and negative areas in the distribution of the matrix components examined. In contrast, areas of CA-positive cell accumulation often possessed a clear margin. According to Provot et al. [27], mRNAs for aggrecan and hyaluronan synthases are not differentially regulated by hypoxia. Thus, the expression of CA may not be connected to that of matrix components via hypoxia in fetal development.

\section{Intermediate filaments and CA9}

We found GFAP-positive cells not only in joint cartilage, but also in tendons and ligaments, albeit in smaller numbers. Thus, GFAP-positive cells seemed to be committed to differentiate into cartilage rather than tendons or ligaments. Miyako et al. [28] previously reported that GFAP-positive cells in the temporomandibular joint disk of young rats drastically increased in number when occlusion between upper and lower molars had commenced. Thus, they considered these cells as key indicators of fibrocartilage maturation of the disk. The GFAP-positive cell distribution was completely included within the CA9-positive cell accumulation area. Therefore, CA9 might be a better marker for differentiation of the enthesis. In this context, we should bear in mind the GFAPpositive mesenchymal cell clusters in which elastic fibers are produced $[29,30]$. CA9 is actually expressed in developing elastic cartilages (auricular, epiglottic and pharyngotympanic tube cartilages) as well as primitive laryngeal ligaments. The latter ligaments are rich in elastic fibers in late stage fetuses [31]. Both CA9 and GFAP might be linked with fibrillin-elastin synthesis. In the present study, desmin and SMA were not found in cartilage, ligaments, or tendons in fetuses. Vimentin immunoreactivity is found not only in osteoprogenitor cells [32,33], but also in mesenchymal tissues in which vasculogenesis will soon occur [34]. In fetuses, the expression pattern of nestin was similar to that of CD34 [35]; both proteins are well known as stem cell markers in adults.

\section{Mechanical stress and CA9: a hypothesis}

Expression of CA9 in cartilage exhibited a rather uniform pattern, as previously reported by Schultz et al. [16]. Positive cells had accumulated in adjacent joint surfaces and the superficial zone at a depth of $0.5-1.0 \mathrm{~mm}$ on the deep side of the joint cavity at 15-16 weeks. At many joints, the expression of CA9 in the cartilage became weaker in larger specimens, but it was maintained through 11-16 weeks. One striking feature of the joint surface was the paired expression of CA9 in the posterior surface of the talus and the flexor digitorum longus tendon; this adjacent topography is fetus-specific 


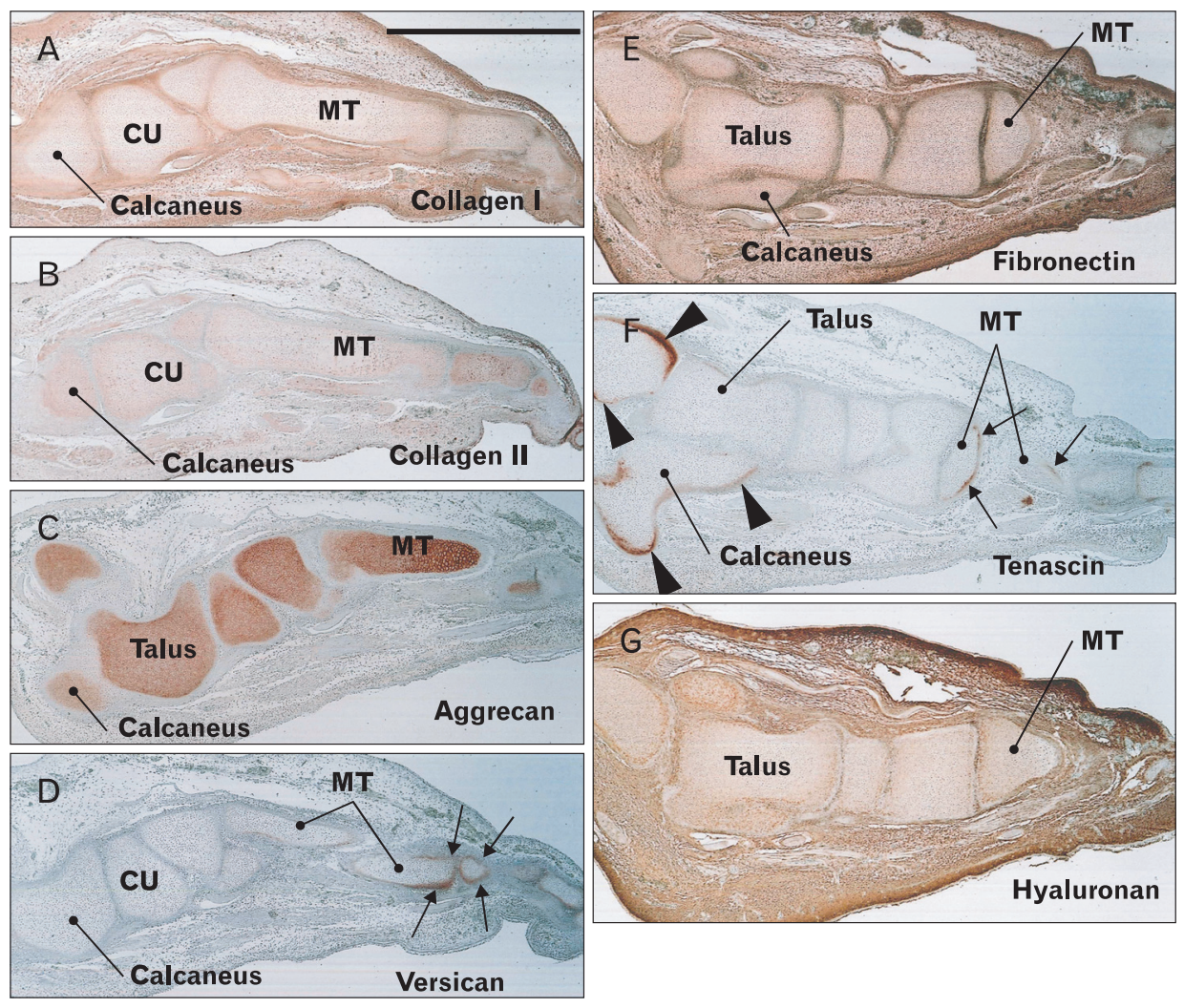

Fig. 7. Expression of matrix components in the foot at 11 weeks. Sagittal sections of the same foot shown in Fig. 2E. Immunohistochemical detection of collagen type 1 (A), collagen type 2 (B), aggrecan $(C)$, versican (D), fibronectin $(E)$, tenascin $(F)$, and hyaluronan $(G)$. Versican and tenascin are expressed in metatarsal bones near the insertion of extensors and flexors. Tenascin is also expressed along the bony surface, but is not detected on the joint surface. The distribution of these matrix components does not appear to correspond with that of CA9 shown in Fig. 2F. CU, cuboid bone; MT, metatarsal bones. Scale bar in panel $(A)=1 \mathrm{~mm}(\mathrm{~A}-\mathrm{G})$.

Table 1. Site-dependent and stage-dependent expressions of CA9 in human fetal development of the enthesis

\begin{tabular}{|c|c|c|c|c|}
\hline & Abdominal wall & $\begin{array}{l}\text { Shoulder } \\
\end{array}$ & Hand and wrist & Foot and ankle \\
\hline 7-8 weeks & Anterior rectus sheath & $\begin{array}{l}\text { Infraspinatus insertion sheath of } \\
\text { biceps tendon }\end{array}$ & $\begin{array}{l}\text { Insertions of FDP, FDS } \\
\text { FCR, ED, ECR }\end{array}$ & Insertion of the peroneus longus \\
\hline 10-12 weeks & Inguinal ligament (Fig. 6F) & $\begin{array}{l}\text { Subscapuralis insertion } \\
\text { glenoid lip (Fig. 2A) }\end{array}$ & Carpal tunnel walls & $\begin{array}{l}\text { Insertions of TP, TA, EDL, FDL } \\
\text { (Fig. 2E) }\end{array}$ \\
\hline $15-16$ weeks & $\begin{array}{l}\text { Linea alba fascia of } \\
\text { gubernaculum }\end{array}$ & $\begin{array}{l}\text { Ligaments to coracoid, } \\
\text { glenoid lip (Fig. 2B) }\end{array}$ & $\begin{array}{l}\text { Scapholunate ligament TFCC, fibrous } \\
\text { sheath of extensors (Fig. 5A) }\end{array}$ & $\begin{array}{l}\text { FDL tendon facing the ankle joint, } \\
\text { retinaculum extensorum (Fig. 2F }\end{array}$ \\
\hline
\end{tabular}

CA9, carbonic anhydrase type IX; FDP, flexor digitorum profundus; FDS, flexor digitorum superficialis; FCR, flexor carpi radialis; ED, extensor digitorum; ECR, extensor carpi radialis; TP, tibialis posterior; TA, tibialis anterior; EDL, extensor digitorum longus; FDL, flexor digitorum longus; TFCC, triangular fibrocartilage complex.

and the two structures are never attached in adults owing to the presence of the joint capsule. In contrast, CA9-positive cells in the enthesis seemed to disappear within 2-3 weeks, and their distribution took on a stochastic quality such that one muscle insertion was positive, whereas another, nearby insertion was negative. One typical example was the shoulder, where the subscapularis muscle insertion was positive and the infraspinatus muscle insertion was negative. Moreover, we often found a positive structure that might not be widely known: the scapholunate interosseus ligament [36]. This structure showed strong expression of CA9 despite the fact that other interosseous carpal ligaments were negative. In- deed, the former develops earliest in the palmar side of the hand at 14 weeks [37].

A subset of the present data on CA9 expression in the enthesis is presented in Table 1, which shows that positive sites were different between smaller fetus (10-12 weeks) and larger fetus (15-16 weeks). CA9 immunoreactivity seemed to first appear slightly after the initial phase of the development of the muscle-associated structure. In our previous studies of fetal anatomy, we hypothesized the appearance of positive sites at 7-8 weeks (Table 1 ). In the abdominal wall, the anterior rectus sheath develops 3-4 weeks earlier than the inguinal ligament and linea alba [38]. Thus, we assumed 
that CA9 was expressed in the rectus sheath at 7-8 weeks. Likewise, in the shoulder, the biceps tendon insertion is formed first, followed by the development of tendon insertions of the supra- and infraspinatus muscles [34]. In the hand and wrist, the long tendons develop first, and the sheath and short muscles appear to develop secondarily [39]. Thus, CA expression was also likely to start first in the long tendons. These site- and stage-dependent aspects of CA9 expression would seem to provide an important signal about the specific physiological status of the enthesis. Was this an adaptation to hypoxia? If hypoxia were the major trigger of CA9 expression, the short duration of immunoreactivity would be difficult to explain because the distinct growth between 10 and 16 weeks should have kept oxygen content low.

Interestingly, the phenotype of CA9-deficient mice is quite mild. We have previously observed gastric hyperplasia and some behavioral changes, but no major abnormalities in the musculoskeletal system of these mice [19]. Likewise, Provot et al. [27] reported that, in mouse limb bud mesenchyme, conditional knockout of hypoxia-inducible factor 1-alpha, which regulates the CA9 gene under hypoxic conditions, does not impair mesenchymal condensation, but alters formation of the cartilaginous primordia. Our interpretation of these authors' model is that there are distinctly impaired developments of the pulley and retinaculum system of the finger and carpal bone arrangement; these relatively minor abnormalities seem to overlap with CA9 accumulation areas in the present study. CA9 expression in joints, ligaments, and tendons may not suggest a critical physiological function of the CA, but instead may signal a specific differentiation status. The contribution of CAs to the development of the musculoskeletal system seems to depend on CAs other than CA9 $[18,40]$.

Is there a status in common among fetal morphologies in which site- and stage-dependent expression of CA9 occurs? We propose that CA9 expression is likely an excellent indicator that the tissues or cells are experiencing temporally restricted mechanical stresses imposed by adjacent structures. At the joint and enthesis, a mechanical stress of one structure one another makes coordinated growth possible; a typical example is the induction of enthesis development by muscle contraction. This is also exemplified by our demonstration that the quadriceps tendon "taught" CA-positive mesenchymal cells at the upper end of the patella. Although it is not widely known, extraocular muscle contractions most likely induce the growth of the conjunctiva via the fetal collateral ligament of the eye [41]. Our findings seem to suggest that the CA9positive fibrous band experiences a mechanical stress. In the fetal joint, the ball and socket were often tightly attached and both expressed CA9. When the ball pushed the ligament or tendon, the latter expressed CA9, as was the case with the annular ligament of the elbow. Consequently, our results suggest that CA9 is an excellent marker of mechanical stress during fetal development of the joint and enthesis.

\section{Study limitation}

Small numbers of the specimens examined were a major limitation of this study, but the solution seemed to be difficult because of unexpected donation. Thus, we were hardly to demonstrate full sets of immunohistochemistry including "all antibodies for all stages." Moreover, beautiful staining was difficult to obtain in smaller specimens (10-12 weeks) partly due to weak expression before establishment of the structures. For CA9, immunohistochemistry, we used not only the original antibody but also the commercial antibody for convenience of readers. However, the back ground especially for striated muscles was rather strong in the commercial one. Because we did not compare between commercial antibodies, we did not consider about the background.

\section{Acknowledgments}

This study was supported by a grant (0620220-1) from the National R \& D Program for Cancer Control, Ministry of Health \& Welfare, Republic of Korea. We are grateful to Dr. Risto Santti, Emeritus Professor at Turku University in Finland, who first organized and encouraged the present research team.

Kim JH analyzed and interpreted the data, drafted the manuscript, and approved the final draft; Parkkilas $S$ performed the planning, analyzed the data, drafted the manuscript, and approved the final draft; Shibata S performed the planning, immunohistochemistry, and approved the final draft; Fujimiya M performed the planning, immunohistochemistry, data analysis, and approved the final draft; Murakami G performed the planning, analyzed the data, drafted the manuscript, and approved the final draft; Cho BH performed the planning, funding, and study conceptualizing, and approved the final draft. 


\section{References}

1. Rot-Nikcevic I, Reddy T, Downing KJ, Belliveau AC, Hallgrímsson B, Hall BK, Kablar B. Myf5-/- :MyoD-/- amyogenic fetuses reveal the importance of early contraction and static loading by striated muscle in mouse skeletogenesis. Dev Genes Evol 2006;216:1-9.

2. Mackey AL, Heinemeier KM, Koskinen SO, Kjaer M. Dynamic adaptation of tendon and muscle connective tissue to mechanical loading. Connect Tissue Res 2008;49:165-8.

3. Kjaer M, Langberg H, Heinemeier K, Bayer ML, Hansen M, Holm L, Doessing S, Kongsgaard M, Krogsgaard MR, Magnusson SP. From mechanical loading to collagen synthesis, structural changes and function in human tendon. Scand J Med Sci Sports 2009;19:500-10.

4. Nowlan NC, Bourdon C, Dumas G, Tajbakhsh S, Prendergast PJ, Murphy P. Developing bones are differentially affected by compromised skeletal muscle formation. Bone 2010;46:1275-85.

5. Milz S, Benjamin M, Putz R. Molecular parameters indicating adaptation to mechanical stress in fibrous connective tissue. Adv Anat Embryol Cell Biol 2005;178:1-71.

6. Magara J, Nozawa-Inoue K, Suzuki A, Kawano Y, Ono K, Nomura S, Maeda T. Alterations in intermediate filaments expression in disc cells from the rat temporomandibular joint following exposure to continuous compressive force. J Anat 2012;220:612-21.

7. Abe S, Rhee SK, Osonoi M, Nakamura T, Cho BH, Murakami G, Ide Y. Expression of intermediate filaments at muscle insertions in human fetuses. J Anat 2010;217:167-73.

8. Yang Y, Makita T. Immunocytochemical colocalization of desmin and vimentin in human fetal skeletal muscle cells. Anat Rec 1996;246:64-70.

9. Kepes JJ, Perentes E. Glial fibrillary acidic protein in chondrocytes of elastic cartilage in the human epiglottis: an immunohistochemical study with polyvalent and monoclonal antibodies. Anat Rec 1988;220:296-9.

10. Viale G, Doglioni C, Dell'Orto P, Zanetti G, Iuzzolino P, Bontempini L, Coggi G. Glial fibrillary acidic protein immunoreactivity in human respiratory tract cartilages and pulmonary chondromatous hamartomas. Am J Pathol 1988;133:363-73.

11. Supuran CT. Carbonic anhydrases: an overview. Curr Pharm Des 2008;14:603-14.

12. Hilvo M, Innocenti A, Monti SM, De Simone G, Supuran CT, Parkkila S. Recent advances in research on the most novel carbonic anhydrases, CA XIII and XV. Curr Pharm Des 2008;14: $672-8$.

13. Gilmour KM. Perspectives on carbonic anhydrase. Comp Biochem Physiol A Mol Integr Physiol 2010;157:193-7.

14. Takacova M, Barathova M, Hulikova A, Ohradanova A, Kopacek J, Parkkila S, Pastorek J, Pastorekova S, Zatovicova M. Hypoxia-inducible expression of the mouse carbonic anhydrase IX demonstrated by new monoclonal antibodies. Int J Oncol 2007;31:1103-10.
15. Barathova M, Takacova M, Holotnakova T, Gibadulinova A, Ohradanova A, Zatovicova M, Hulikova A, Kopacek J, Parkkila S, Supuran CT, Pastorekova S, Pastorek J. Alternative splicing variant of the hypoxia marker carbonic anhydrase IX expressed independently of hypoxia and tumour phenotype. Br J Cancer 2008;98:129-36.

16. Schultz M, Jin W, Waheed A, Moed BR, Sly W, Zhang Z. Expression profile of carbonic anhydrases in articular cartilage. Histochem Cell Biol 2011;136:145-51.

17. Liao SY, Lerman MI, Stanbridge EJ. Expression of transmembrane carbonic anhydrases, CAIX and CAXII, in human development. BMC Dev Biol 2009;9:22.

18. Scheibe RJ, Mundhenk K, Becker T, Hallerdei J, Waheed A, Shah GN, Sly WS, Gros G, Wetzel P. Carbonic anhydrases IV and IX: subcellular localization and functional role in mouse skeletal muscle. Am J Physiol Cell Physiol 2008;294:C402-12.

19. Gut MO, Parkkila S, Vernerová Z, Rohde E, Závada J, Höcker M, Pastorek J, Karttunen T, Gibadulinová A, Závadová Z, Knobeloch KP, Wiedenmann B, Svoboda J, Horak I, Pastoreková S. Gastric hyperplasia in mice with targeted disruption of the carbonic anhydrase gene Car9. Gastroenterology 2002;123:1889903.

20. Hayashi S, Murakami G, Ohtsuka A, Itoh M, Nakano T, Fukuzawa Y. Connective tissue configuration in the human liver hilar region with special reference to the liver capsule and vascular sheath. J Hepatobiliary Pancreat Surg 2008;15:640-7.

21. Kawase T, Shibata S, Katori Y, Ohtsuka A, Murakami G, Fujimiya M. Elastic fiber-mediated enthesis in the human middle ear. J Anat 2012;221:331-40.

22. Shibata S, Fukada K, Imai H, Abe T, Yamashita Y. In situ hybridization and immunohistochemistry of versican, aggrecan and link protein, and histochemistry of hyaluronan in the developing mouse limb bud cartilage. J Anat 2003;203:425-32.

23. Nakasone A, Shibata S, Suzuki S, Yamashita Y, Ohyama K. Laser burn wound healing in naso-labial region of fetal and neonatal mice. Oral Dis 2007;13:45-50.

24. Yokohama-Tamaki T, Maeda T, Tanaka TS, Shibata S. Functional analysis of CTRP3/cartducin in Meckel's cartilage and developing condylar cartilage in the fetal mouse mandible. J Anat 2011;218:517-33.

25. Palmer AK. Triangular fibrocartilage complex lesions: a classification. J Hand Surg Am 1989;14:594-606.

26. Nam YS, Han SH, Shin SY. Detailed anatomy of the capsulopalpebral fascia. Clin Anat 2012;25:709-13.

27. Provot S, Zinyk D, Gunes Y, Kathri R, Le Q, Kronenberg HM, Johnson RS, Longaker MT, Giaccia AJ, Schipani E. Hif-1alpha regulates differentiation of limb bud mesenchyme and joint development. J Cell Biol 2007;177:451-64.

28. Miyako H, Suzuki A, Nozawa-Inoue K, Magara J, Kawano Y, Ono K, Maeda T. Phenotypes of articular disc cells in the rat temporomandibular joint as demonstrated by immunohistochemistry for nestin and GFAP. J Anat 2011;219:472-80.

29. Katori Y, Takeuchi H, Rodríguez-Vázquez JF, Kitano H, Murakami G, Kawase T. Fetal development of the human epig- 
lottis revisited: appearance of GFAP-positive mesenchymal cells and fibrous connections with other laryngeal and lingual structures. Ann Anat 2011;193:149-55.

30. Ikari Y, Katori Y, Ohtsuka A, Rodríguez-Vázquez JF, Abe H, Kawase T, Murakami G, Abe S. Fetal development and variations in the cartilages surrounding the human external acoustic meatus. Ann Anat 2013;195:128-36.

31. Kinoshita H, Umezawa T, Omine Y, Kasahara M, RodríguezVázquez JF, Murakami G, Abe S. Distribution of elastic fibers in the head and neck: a histological study using late-stage human fetuses. Anat Cell Biol 2013;46:39-48.

32. Watanabe H, Miake K, Sasaki J. Immunohistochemical study of the cytoskeleton of osteoblasts in the rat calvaria. Intermediate filaments and microfilaments as demonstrated by detergent perfusion. Acta Anat (Basel) 1993;147:14-23.

33. Shapiro F, Cahill C, Malatantis G, Nayak RC. Transmission electron microscopic demonstration of vimentin in rat osteoblast and osteocyte cell bodies and processes using the immunogold technique. Anat Rec 1995;241:39-48.

34. Abe S, Nakamura T, Rodriguez-Vazquez JF, Murakami G, Ide Y. Early fetal development of the rotator interval region of the shoulder with special reference to topographical relationships among related tendons and ligaments. Surg Radiol Anat 2011;33:609-15.

35. Abe S, Suzuki M, Cho KH, Murakami G, Cho BH, Ide Y. CD34- positive developing vessels and other structures in human fetuses: an immunohistochemical study. Surg Radiol Anat 2011;33:919-27.

36. Berger RA, Kauer JM, Landsmeer JM. Radioscapholunate ligament: a gross anatomic and histologic study of fetal and adult wrists. J Hand Surg Am 1991;16:350-5.

37. Mérida-Velasco JA, Garcia-Garcia JD, Espín-Ferra J, SánchezMontesinos I. Development of the human wrist joint ligaments. Anat Rec 1996;245:114-21.

38. Yang JD, Hwang HP, Kim JH, Rodríguez-Vázquez JF, Abe S, Murakami G, Cho BH. Development of the rectus abdominis and its sheath in the human fetus. Yonsei Med J 2012;53:102835.

39. Cho KH, Kim JH, Ha YS, Murakami G, Cho BH, Abe S. Development of the deep flexor tendons and lumbricalis muscle in the hand and foot: a histological study using human mid-term foetuses. Folia Morphol (Warsz) 2012;71:154-63.

40. Väänänen HK. Immunohistochemical localization of carbonic anhydrase isoenzymes I and II in human bone, cartilage and giant cell tumor. Histochemistry 1984;81:485-7.

41. Osanai H, Rodríguez-Vázquez JF, Abe H, Murakami G, Ohguro $\mathrm{H}$, Fujimiya M. Fetal check ligament connected between the conjunctiva and the medial and lateral recti. Invest Ophthalmol Vis Sci 2011;52:7175-9. 